Proceedings

\title{
Preliminary Study on Electrochemical Ion Imprinted Polymeric Film in Sensor Development for Cd(II) Ions Determination in Water ${ }^{+}$
}

\author{
Sabrina Di Masi ${ }^{1, *}$, Antonio Pennetta ${ }^{2}$ and Cosimino Malitesta ${ }^{1}$ \\ 1 Laboratorio di Chimica Analitica, Dipartimento di Scienze e Tecnologie Biologiche ed Ambientali, \\ Università del Salento, Via Monteroni, 73100 Lecce, Italy; \\ 2 Laboratorio di Spettrometria di Massa Analitica e Isotopica, Dipartimento di Beni Culturali, Università del \\ Salento, Via Monteroni, 73100 Lecce, Italy; \\ * Correspondence: Tel.: + 39-0832-299-457 \\ + Presented at the 1st International Electronic Conference on Biosensors, 2-17 November 2020. \\ Received: date; Accepted: date; Published: date
}

\begin{abstract}
Preliminary results on an electrosynthesised ion imprinted polymeric film (IIP-film) for $\mathrm{Cd}(\mathrm{II})$ ions determination in sensor development are here reported. The sensor was prepared by electropolymerisation of 4-aminophenylacetic acid (4-APA) monomer in presence of $\mathrm{Cd}(\mathrm{II})$ ions, which acts as the template. The screen printed carbon electrodes (SPCE) were used as transducer during sensor development, whereas the cyclic voltammetry (CV) and differential pulse voltammetry (DPV) were selected as the electrochemical methods for the synthesis and Cd(II) ions sensing, respectively. The incubation of the developed sensor in $\mathrm{NaOH} 250 \mathrm{mM}$ involved into remove the template and the formation of specific recognition cavities into the polymer. A multivariate optimisation based on central composite design (CCD) was employed to study the effect of three independent parameters on electrochemical performances of the sensor. The electrochemical characterisation of sensors was performed in ferrocyanide-ferricyanide redox couple and in $\mathrm{KCl} 0.1 \mathrm{M}$, the latter revealing redox properties from the polymeric film. The performances of sensors and the control (non-imprinted film, NIP) was observed in sodium acetate buffer $(100 \mathrm{mM}, \mathrm{pH}=5)$ over the $\mathrm{Cd}(\mathrm{II})$ concentration range $0.1-10 \mu \mathrm{M}$.
\end{abstract}

Keywords: ion imprinted polymer; 4-APA; electrochemical sensor; $\mathrm{Cd}(\mathrm{II})$ ions; electropolymerisation

\section{Introduction}

Heavy metals pollution refers to global issue, due to the high toxicity and dangerous effects on environment and human health. Among heavy metals, cadmium is one of the most toxic. Main sources of this ion in environment is industrial wastewater, fertilizers and so on. Currently, the most analytical method for cadmium detection is represented by atomic adsorption spectroscopy (AAS), inductively coupled plasma-mass spectroscopy (ICP-MS) and inductively coupled plasma atomic emission spectrometry (ICP-AES). Those techniques are sensitive, accurate but also expensive, and on-site determination of targets is not so suitable. Because the complexity of those instrumentation, there is the need to point different methods to be available for on-site determination. Electrochemical methods can be used for that. Moreover, diverse electrochemical methods are today explored for the determination of heavy metals in water environment [1].

Imprinted polymers define robust and artificial materials able to mimic recognition processes of such analytes, such as proteins, small molecules, or ions [2]. The process results in the selective formation of ion-sized imprinted cavities, which are complementary to a specific template in terms 
of its functional groups. These materials can be easily applied to identify, monitor and remove the target ions in water environment [3]. In this view, the ion imprinted polymers (IIPs) can be described. Their synthesis can be carried out both chemically and electrochemically. The latter leads to the preparation of imprinted films, which are compatible in conjunction with transducers in sensor development $[4,5]$. Very few works report the electrochemical synthesis of ion imprinted polymers and their application as sensors for metal ion detection [6,7]. With this regard, we propose the synthesis, optimisation, characterisation and subsequent application of an electrosynthesised IIPs for the electrochemical detection of cadmium (II) in water. The proposed sensor was prepared by electropolymerisation of 4-aminophenylacetic acid (4-APA) monomer in presence of Cd(II) ions, which acts as the template. The screen printed carbon electrodes (SPCE) were used as transducer during sensor development, whereas the cyclic voltammetry $(\mathrm{CV})$ and differential pulse voltammetry (DPV) were selected as the electrochemical methods for the synthesis and Cd(II) ions sensing, respectively. The incubation of the developed sensor in $\mathrm{NaOH} 250 \mathrm{mM}$ involved into remove the template and the formation of specific recognition cavities into the polymer. A multivariate optimisation was employed for studying the effect of three independent parameters on electrochemical performances of the sensor. The electrochemical characterisation of sensors was performed in ferrocyanide-ferricyanide redox couple and in $\mathrm{KCl} 0.1 \mathrm{M}$, the latter revealing redox properties from the polymeric film. The performances of sensors and the control (NIP) was observed in sodium acetate buffer $(100 \mathrm{mM}, \mathrm{pH}=5)$ over the $\mathrm{Cd}(\mathrm{II})$ concentration range of $0.1-10 \mu \mathrm{M}$.

\section{Materials and Methods}

\subsection{Materials}

Acetic acid, 4-Aminophenylacetic acid (4-APA, 98\%), sodium acetate trihydrate, cadmium nitrate tetrahydrate $(98 \%)$, ethylenediaminetetraacetic acid $(99 \%)$ were purchased from SigmaAldrich (Italy). Sulphuric acid and sodium hydroxide solutions were commercially available as analytical reagent grade. All reagents were used without further purification. MilliQ water was used for washing the polymeric film after the preparation. Sodium acetate buffer $(100 \mathrm{mM}, \mathrm{pH}=5)$.

\subsection{Apparatus}

CV and DPV measurements were performed using a PalmSens potentiostat equipped with a cable connector (DropSens, Italy) for screen-printed electrodes. PSTrace was the software to control the instrument and data acquisition. The polymeric film was deposited on screen-printed carbon electrode (SPCE). The SPCEs were composed of three-electrode configuration on a planar ceramic support $(3.3 \times 1 \mathrm{~cm})$ and they consisted of a carbon disk-shaped working electrode (4 mm diameter), a platinum electrode as counter electrode and a pseudo $\mathrm{Ag} / \mathrm{AgCl}$ paste electrode as reference electrode. SPCE were commercially available (Metrohm, Italy).

\subsection{Preparation of Electrosynthesised Ion Imprinted Polymer and Non-Imprinted Polymer}

The preparation of ion imprinted polymer (IIP) based on poly-4-aminophenylacetic (poly-4APA) films was performed by cyclic voltammetry $(\mathrm{CV})$ in a potential range between -0.2 and $1.2 \mathrm{~V}$ vs. pseudo $\mathrm{Ag} / \mathrm{AgCl}$, at a scan rate of $50 \mathrm{mV} \mathrm{s}^{-1}$ for 40 cycles in a solution of $\mathrm{H}_{2} \mathrm{SO}_{4} 0.5 \mathrm{M}$ containing $1 \mathrm{mM}$ of $\mathrm{Cd}^{2+}$ ions. The porogen was chosen based on previous works about the electrosynthesis of poly-4-APA on SPE [8]. After the electropolymerisation, the electrode was rinsed with MilliQ water and incubated in different solvent (EDTA $100 \mathrm{mM}$ and $250 \mathrm{mM}, \mathrm{H}_{2} \mathrm{SO}_{4} 500 \mathrm{mM}, \mathrm{NaOH} 100 \mathrm{mM}$ and $250 \mathrm{mM}$ ) to remove the target. The preparation of the control (non-imprinted polymer, NIP) was obtained with the same protocol, but without adding the template into the polymerisation mixture. The treatment in $\mathrm{NaOH} 250 \mathrm{mM}$ was also performed on NIP. All prepared sensors were taken in air when not in use.

\section{4. $C d^{2+}$ Ion Sensing}


The electrochemical responses of IIP and NIP films towards $\mathrm{Cd}^{2+}$ ions were recorded using DPV measurements in the potential range of -0.2 to $+0.4 \mathrm{~V}$, modulation amplitude of $50 \mathrm{mV}$, step potential of $4.95 \mathrm{mV}$, and equilibration time of $2 \mathrm{~s} . \mathrm{Cd}^{2+}$ ions interacted with the imprinted film by drop-casting on the electrode surface an appropriate amount $(100 \mu \mathrm{L})$ of a solution of sodium acetate buffer (100 $\mathrm{mM}, \mathrm{pH}=5)$ containing different concentration of $\mathrm{Cd}^{2+}$ ions $(0.1-10 \mu \mathrm{M})$, by leaving the drop on the electrode for $10 \mathrm{~min}$. After each measurement, the electrode surface was gently washed with sodium acetate buffer for $2 \mathrm{~min}$.

\subsection{Experimental Design in Optimisation Studies}

Multivariate optimisation was conducted with the light to optimise the development of IIPs and NIP. The selected optimisation model was the central composite design (CCD), which allowed the selection of main three factors affecting the development of the sensors, such as (i) the monomer concentration, (ii) the rate between template-monomer (mainly affecting the number of cavities on the polymeric network) and (iii) the number of CV cycles during the electrosynthesis. MODDE (version 12) was used for design, mathematical modelling and optimisation. The levels of studied independent variables are listed in Table 1.

Table 1. Levels of independent variables considered in this work.

\begin{tabular}{ccc}
\hline Variable & Low & High \\
\hline Monomer concentration $\left(\mathrm{X}_{1}\right)$ & 0.5 & 5 \\
Rate $\mathrm{Cd}^{2+}$ /monomer $\left(\mathrm{X}_{2}\right)$ & 1 & 3 \\
Number of CV cycles $\left(\mathrm{X}_{3}\right)$ & 10 & 40 \\
\hline
\end{tabular}

The response was the difference of current $(\Delta i, \mu \mathrm{A})$ recorded in ferrocyanide-ferricyanide redox probe before and after the electropolymerisation of the different imprinted sensors. Based on CCD principle, the design consisted of $2^{\mathrm{k}}$ fractional factorial points plus $2^{\mathrm{k}}$ axial points and 1 center point, where $\mathrm{k}$ defines the number of central points (in this case, $\mathrm{k}=3$ ). 18 experiments run were conducted, and the second order polynomial equation consisted of linear, quadratic and first order interaction terms is shown below (Equation (1)):

$$
\mathrm{Y}=\beta_{0}+\sum_{i=1}^{k} \beta_{i} X_{i}+\sum_{i=1}^{k} \beta_{i i} X_{i}^{2}+\sum_{i=1}^{k} \sum_{j \neq i)}^{k} \beta_{i j} X_{i} X_{j}+\varepsilon,
$$

where $\mathrm{Y}$ is the response variables, $\mathrm{X}_{i}$ represent the dependent variables, $\beta_{0}, \beta_{i}, \beta_{i i}, \beta_{i j}$ were the regression coefficient for intercept, linear, quadratic and interaction terms, respectively.

\section{Results and Discussions}

The electropolymerisation of 4-APA in presence of $\mathrm{Cd}^{2+}$ produced a sensitive polymeric imprinted film for that template, showing superior characteristics against its control. The optimal condition of synthesis was established by employing a multivariate experimental design, which approach is currently gained interest by scientists to optimised sensors and biomimetic sensors. The advantage of using the produced IIPs consisted on revealing a redox property of the polymer, directly addresses on the interaction between imprinted cavities and template. The interaction was visible close to $+0 \mathrm{~V}$ (see related DPV measurements), which potential is quite high than normally observed for the electroactivity of $\mathrm{Cd}^{2+}$ in solution. 


\subsection{Preparation of Electrosynthesised IIP and NIP Films}

Figure 1a presented a typical cyclic voltammetry recorded during the electropolymerisation of $2.1 \mathrm{mM}$ 4-APA in presence of $2.1 \mathrm{mM} \mathrm{Cd}^{2+}$ ions in $0.5 \mathrm{M} \mathrm{H}_{2} \mathrm{SO}_{4}$ on screen printed carbon electrode. Figure $1 \mathrm{~b}$ shows the electropolymerisation of 4-APA on SPCE, without the template (NIP).

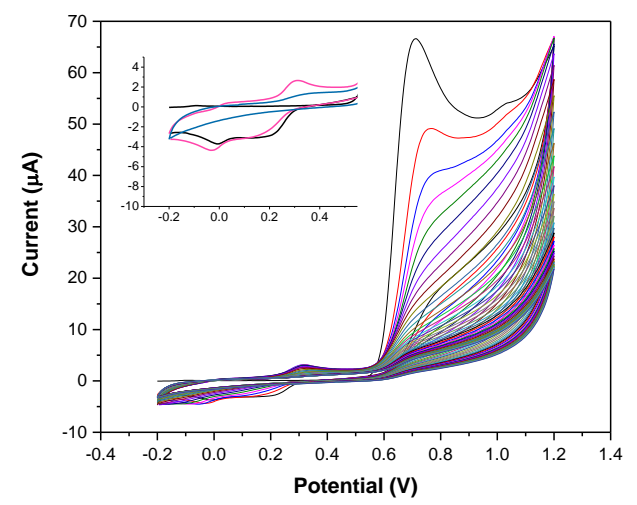

(a)

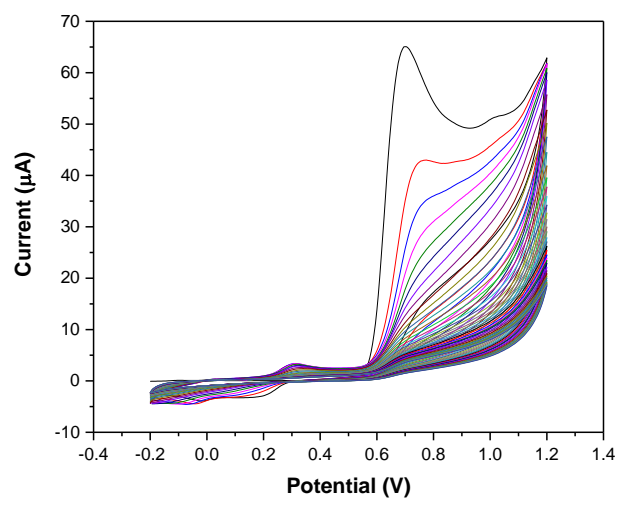

(b)

Figure 1. (a) The electropolymerisation of $2.1 \mathrm{mM} 4$-APA in presence of $2.1 \mathrm{mM} \mathrm{Cd}^{2+}$ in $0.5 \mathrm{M} \mathrm{H}_{2} \mathrm{SO}_{4}$. Inset: Focused CV for (black) 1th, (pink) 2nd, (blue) 17th cycle; (b) The electropolymerisation of 2.1 $\mathrm{mM} 4-\mathrm{APA}$ in $0.5 \mathrm{M} \mathrm{H}_{2} \mathrm{SO}_{4}$. Voltammetric condition: (i) potential range: -0.2 to $+1.2 \mathrm{~V}$; (ii) scan rate: $50 \mathrm{mV} \mathrm{s}^{-1}$; (iii) CV cycles: 40.

During the $\mathrm{CV}$, the first peak at $+0.70 \mathrm{~V}$ indicated the formation of cation radicals that promoted the polymerisation process, once to the oxidation of 4-APA. Further peaks at $+0.19 \mathrm{~V}$ and at $-0.025 \mathrm{~V}$ are related to the reduction of the polymer film on the SPCE surface. Following the second potential cycle, two oxidation waves appeared at the potentials of +0.040 and $+0.305 \mathrm{~V}$, corresponding to the oxidation of the formed polymeric film. After around 17 cycles of polymerisation, it was notable a decrease in the anodic peaks current, indicating the subsequent formation of the polymer film (see Inset of Figure 1a). Finally, the formation of the film produced a partial blockage of the electrode surface. The electropolymerisation of NIP (Figure 1b) followed the same interpretation of the process, with differences in terms of current appeared along the second cycle of $\mathrm{CV}$.

\subsection{Optmisation of Sensor Performances by Experimental Design}

The optimisation of performances was possible by a multivariate approach, that considered all factors together, including linear, quadratic and interaction terms in the model. All the selected factors were related on the electrosynthesis process. Among them, with the emphasis to develop imprinted materials, the relationship between all reagent should be described. Preliminary results shown the factor's importance on responses were the initial concentration of functional monomer $\left(X_{1}\right)$ and the number of $C V$ cycles during the electrosynthesis $\left(X_{3}\right)$. Figure 2 shows the significant coefficients related to factors. 


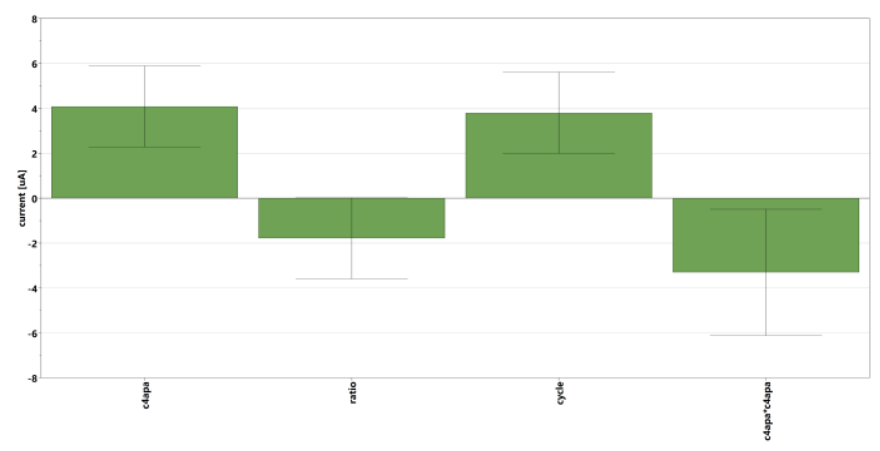

Figure 2. Plot of the significant coefficients obtained from model.

The regression equation for the achieved responses, including significant factors, is reported (Equation (2)).

$$
\mathrm{Y}=14.86+4.08 X_{1}-1.78 X_{2}+3.80 X_{3}-3.30 X_{1} X_{1}
$$

After evaluation of the Equation (2), it appears as the monomer concentration is in strong correlation with the other factors. In particular the ratio of monomer/ $\mathrm{Cd}^{2+}$ should be regulated to assume a correct orientation of cavities on the polymer network. The factor related on the grown of the electrosynthesised imprinted film was also significant, confirming as the deposition of the film on electrode surface is involved in the difference of currents recorded by the electrochemical probe. With the light to maximise the responses, the experimental conditions used for further measurements were (i) $2.1 \mathrm{mM}$ 4-APA, (ii) $2.1 \mathrm{mM} \mathrm{Cd}^{2+}$ (ratio 1:1), and (iii) $40 \mathrm{CV}$ cycles during the electrosynthesis.

\subsection{Electrochemical Characterisation of IIP and NIP Films}

The prepared sensors were first subjected to electrochemical characterisation in ferrocyanideferricyanide redox probe and in $\mathrm{KCl} 0.1 \mathrm{M}$, by applying a CV measurement for bare SPCE, IIP film and NIP film after the electrodeposition (Figure 3a,b).

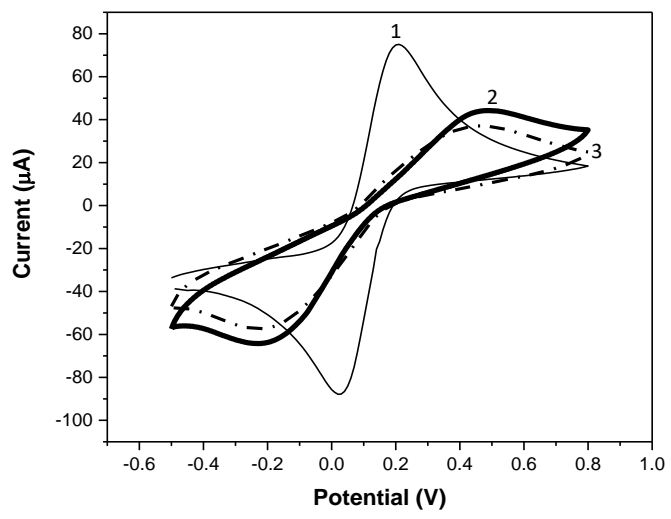

(a)

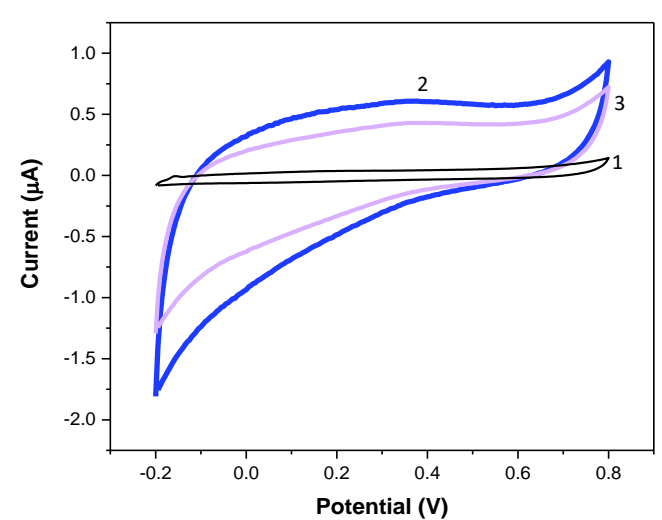

(b)

Figure 3. (a) Electrochemical characterisation by CV (5th cycle) in ferrocyanide-ferricyanide redox probe for (1) bare screen-printed carbon electrode; (2) $\mathrm{Cd}^{2+}$-IIP film/SPCE and (3) NIP film/SPCE after polymerisation. Voltammetric condition: (i) potential range: -0.5 to $+0.8 \mathrm{~V}$; (ii) scan rate: $50 \mathrm{mV} \mathrm{s}^{-1}$; (iii) CV cycles: 5; (b) Electrochemical characterisation by CV (5th cycle) in $0.1 \mathrm{M} \mathrm{KCl}$ for (1) bare screen printed carbon electrode; (2) $\mathrm{Cd}^{2+}$-IIP film/SPCE and (3) NIP film/SPCE after polymerisation. Voltammetric condition: (i) potential range: -0.2 to $+0.8 \mathrm{~V}$; (ii) scan rate: $50 \mathrm{mV} \mathrm{s}{ }^{-1}$; (iii) CV cycles: 5 . 
Both electrochemical characterisation revealed higher electroactivity of the imprinted polymer when compared to NIP film. In addition, as shown in Figure $2 b$, no signals were obtained for bare SPCE. The electroactivity of IIP film than NIP suggest the imprinting effect of the polymer, where possibly $\mathrm{Cd}^{2+}$ ions is able to enhance the overall electrochemical process during polymerisation.

The removal of the template ion - to obtain the imprinted cavities - was carried out by exposure of the sensor to different solutions, such as EDTA $100 \mathrm{mM}$ and $250 \mathrm{mM}, \mathrm{H}_{2} \mathrm{SO}_{4} 500 \mathrm{mM}, \mathrm{NaOH} 100$ $\mathrm{mM}$ and $250 \mathrm{mM}$. In all cases, different times of elution were tested, in a range between 1 and $15 \mathrm{~min}$ $(1,3,5,10,15 \mathrm{~min}$, respectively). As the most effective method, $\mathrm{NaOH} 250 \mathrm{mM}$ incubated for $3 \mathrm{~min}$ was used. CV markable differences recorded in $\mathrm{KCl} 0.1 \mathrm{M}$ for NIP and IIPs treated with $\mathrm{NaOH} 250$ $\mathrm{mM}$ were visible (Figure 4), confirming the elution of $\mathrm{Cd}^{2+}$ ions from the imprinted cavities.

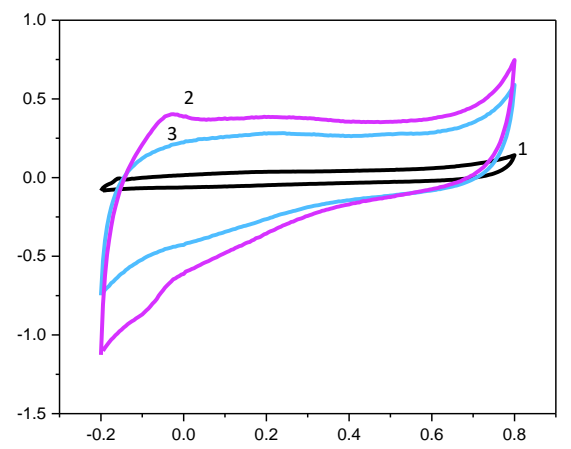

Figure 4. Electrochemical characterisation by $\mathrm{CV}$ (5th cycle) in $0.1 \mathrm{M} \mathrm{KCl}$ for (1) bare screen printed carbon electrode; (2) $\mathrm{Cd}^{2+}$-IIP film/SPCE and (3) NIP film/SPCE after treatment in $250 \mathrm{mM} \mathrm{NaOH}$ for 3 min.. Voltammetric condition: (i) potential range: -0.2 to $+0.8 \mathrm{~V}$; (ii) scan rate: $50 \mathrm{mV} \mathrm{s}^{-1}$; (iii) $\mathrm{CV}$ cycles: 5 .

\subsection{Electrochemical Performances of IIP and NIP Film}

The electrochemical sensing of $\mathrm{Cd}^{2+}$ ions was performed by DPV measurements on NIP and $\mathrm{Cd}^{2+}$-IIP film. $100 \mathrm{mM}$ sodium acetate buffer $(\mathrm{pH}=5)$ was selected as the electrolyte solution for the determination of $\mathrm{Cu}^{2+}$ ions. DPV measurements recorded for $\mathrm{Cd}^{2+}$ IIIP film are shown in Figure 5, and related calibration curves are also reported (Figure $5 b$ ).

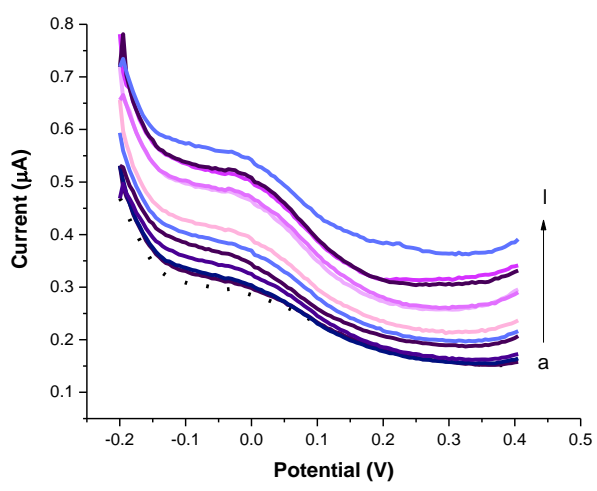

(a)

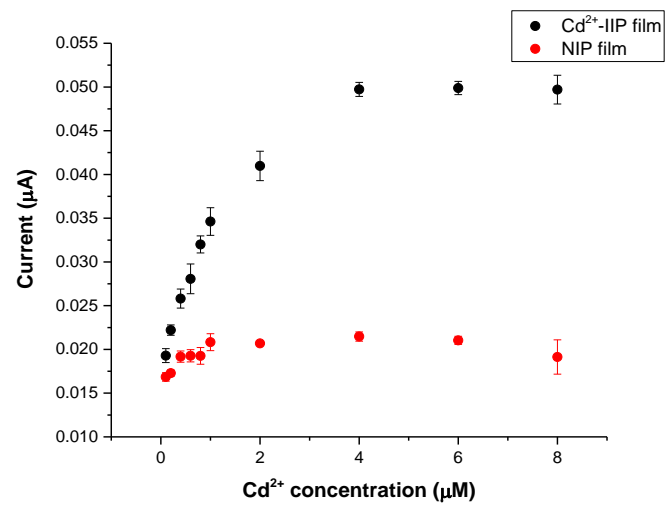

(b)

Figure 5. (a) Differential pulse voltammograms recorded for $\mathrm{Cd}^{2+}$-IIP film after the exposure to (a) blank (sodium acetate buffer), (b) 0.1, (c) 0.2, (d) 0.4, (e) 0.6, (f) 0.8, (g) 1.0, (h) 2.0, (i) 4.0, (j) 6.0, (k) 8.0, (l) $10 \mu \mathrm{M}$ of $\mathrm{Cd}^{2+}$ ions in presence of sodium acetate buffer; (b) Comparison of the electrochemical responses between $\mathrm{Cd}^{2+}$-IIP and NIP films along all tested $\mathrm{Cd}^{2+}$ concentration; (c) Comparison 
between responses from $\mathrm{Cd}^{2+}$-IIP and NIP film in the linear range revealed between 0.1 and $1 \mu \mathrm{M} \mathrm{Cd}^{2+}$ ions.

As shown from Figure 5a, the current responses value increased within the tested $\mathrm{Cd}^{2+}$ ion concentration. However, saturation reached value upper than $2 \mu \mathrm{M}$, due to the occupancy of cavities. Notably, the imprinted sensor shows high affinity and specificity towards $\mathrm{Cd}^{2+}$ ions than that obtained for NIP films, confirming the imprinting effect on this polymer. The linear regression was established between 0.1 and $1 \mu \mathrm{M}$, with a sensitivity of $0.0163 \mu \mathrm{A} \mu \mathrm{M}^{-1}$. In addition, it was possible to evaluate the imprinted factor as 6.86 , highly indicating the specific recognition of template from imprinted cavities on $\mathrm{Cd}^{2+}$ IIP films.

The proposed imprinted sensor shows high sensitivity and posses superior specific properties towards $\mathrm{Cd}^{2+}$ ions. This preliminary results are currently encouraged us to perfom further experiments in regard to selectivity properties of the imprinted polymer against NIP and its application to real water matrices, which discussion will be presented soon.

\section{Conclusions}

Preliminary study on electrosynthesis of ion imprinted polymeric sensor on SPCE transducer for $\mathrm{Cd}^{2+}$ ion determination in water is here reported. The electrosynthesis of the imprinted cavities revealed the newly approach to produce highly sensitive films towards environmental targets. In this light, the developed imprinted polymeric film shows greater sensitivity than NIP film, with an imprinting factor of 6.86. Those achieved preliminary results open the possibly to employ this sensor for quantitative determination of $\mathrm{Cd}^{2+}$ ions in water. Further experiments to evaluate more properties of the sensor are currently under study.

Funding: This research was funded by the project "CASCADE" (014-2020 Interreg V-A IT-HR CBC "trategic" project ID 10255941).

Acknowledgments: Authors would like to thank Marco Costa for his helping during experiments.

Conflicts of Interest: The authors declare no conflict of interest.

\section{References}

1. Wong, A.; A. Ferreira, P.; Santos, A.M.; Cincotto, F.H.; Silva, R.A.B.; Sotomayor, M.D.P.T. A new electrochemical sensor based on eco-friendly chemistry for the simultaneous determination of toxic trace elements. Microchem. J. 2020, 158, 105292, doi:10.1016/j.microc.2020.105292.

2. Li, N.; Yang, H. Construction of natural polymeric imprinted materials and their applications in water treatment: A review. J. Hazard. Mater. 2021, 403, doi:10.1016/j.jhazmat.2020.123643.

3. Jinadasa, K.K.; Peña-Vázquez, E.; Bermejo-Barrera, P.; Moreda-Piñeiro, A. New adsorbents based on imprinted polymers and composite nanomaterials for arsenic and mercury screening/speciation: A review. Microchem. J. 2020, 156, 104886, doi:10.1016/j.microc.2020.104886.

4. Crapnell, R.D.; Hudson, A.; Foster, C.W.; Eersels, K.; van Grinsven, B.; Cleij, T.J.; Banks, C.E.; Peeters, M. Recent advances in electrosynthesized molecularly imprinted polymer sensing platforms for bioanalyte detection. Sensors 2019, 19, 1204, doi:10.3390/s19051204.

5. Sharma, P.S.; Pietrzyk-Le, A.; D'Souza, F.; Kutner, W. Electrochemically synthesized polymers in molecular imprinting for chemical sensing. Anal. Bioanal. Chem. 2012, 402, 3177-3204, doi:10.1007/s00216-011-5696-6.

6. Sharma, G.; Kandasubramanian, B. Molecularly Imprinted Polymers for Selective Recognition and Extraction of Heavy Metal Ions and Toxic Dyes. J. Chem. Eng. Data 2020, 65, 396-418, doi:10.1021/acs.jced.9b00953.

7. Di Masi, S.; Pennetta, A.; Guerreiro, A.; Canfarotta, F.; Egidio, G.; Benedetto, D.; Malitesta, C. Sensor based on electrosynthesised imprinted polymeric film for rapid and trace detection of copper (II) ions. Sensors Actuators B. Chem. 2020, 307, 127648, doi:10.1016/j.snb.2019.127648.

8. Pimenta, T.C.; Santos, C.d.C.; Thomasini, R.L.; Ferreira, L.F. Impedimetric immunosensor for dengue diagnosis using graphite screen-printed electrodes coated with poly(4-aminophenylacetic acid). Biomed. Microdevices 2018, 20, 1-9, doi:10.1007/s10544-018-0324-2. 
Publisher's Note: MDPI stays neutral with regard to jurisdictional claims in published maps and institutional affiliations.

(C) 2020 by the authors. Submitted for possible open access publication under the terms and conditions of the Creative Commons Attribution (CC BY) license (http://creativecommons.org/licenses/by/4.0/). 